\title{
CONSIDÉRATIONS ANTHROPOLOGIQUES CONCERNANT \\ LA POPULATION DU MOYEN AGE DE VADU ANEI
}

Laura Georgescu

\section{CONSIDERAȚiI ANTROPOLOGICE CU PRIVIRE LA POPUlația MEdiEVALĂ DE LA VADU ANEI}

\section{Rezumat}

Autoarea prezintă considerații antropologice pe baza studiului unui lot de subiecți descoperiți în necropola medievală de la Vadu-Anei în comparație cu subiecții din cuprinsul altor necropole medievale din Câmpia Română cu care au tangență din punct de vedere al analizei antropologice.

Din cele 446 de morminte cercetate a fost ales un lot de 140 de subiecți, a căror stare de conservare a permis un studiu de detaliu. Analiza lor s-a făcut pe baza caracteristicilor metrice şi morfologice pentru încadrarea tipologică. Sub raportul metric şi morfologic, seria subiecților din necropola de la Vadu-Anei este polimorfă.

Studiul morfofuncțional a relevat prezența unor impresiuni musculare puternice la nivelul inserțiilor musculare, fapt care ne permite să avansăm ipoteza că subiecții de la Vadu Anei erau destul de omogeni ca grup, cu o activitate intensă, caracteristică populației agricole din zona cercetată. De asemenea s-a putut diagnostica prezența maladiei reumatismale, cu evoluțiile ei deformante, datorită mediului umed, a fracturilor consolidate defectuos şi deformant datorită unei practici empirice, sau în unele cazuri fără nici un fel de intervenție reparatorie.

S-a constatat frecvența foarte mare a mortalității infantile, în special la grupa infans I, a adolescenţilor şi a femeilor din grupa de vârstă foarte tânără şi tânără nu poate fi întâmplătoare. De exemplu, gruparea copiilor în anumite zone poate fi datorită unor epidemii, care bineînțeles a afectat nu numai această grupă de vârstă, dar şi cea a femeilor foarte tinere.

În privința speranței de viață, distribuirea populației pe grupe de vârstă ne redă mai amănunțit structura unei populații sau a unui grup uman. În acest fel ne dăm seama dacă populația e formată mai ales din elemente tinere şi adulte şi cât la sută din elemente bătrâne, inversând rezultatele mortalităţii constatate, deoarece inhumații nu reprezintă totalitatea indivizilor care au viețuit în arealul studiat.

O altă problemă pe care o ridică grupul uman de la Vadu Anei este aceea a depunerii inhumaților: ei sunt depuşi în decubit dorsal cu membrele superioare flectate pe bazin, pe centură sau cu ambele membre superioare (sau numai cu unul din ele) pe claviculă, iar în unele cazuri pe stern. Reprezentanții acestor moduri de înhumare au caractere comune-pot fi diferențe regionale de concepție. Acest fapt, ca şi la Măicăneşti-Străuleşti, nu exclude posibilitatea originilor etnice diferite, cunoscut fiind faptul că elementele mediteraneene au o zonă de difuziune foarte largă.

Cuvinte cheie: necropolă medievală

Mots clef: nécropole médiévale

Les recherches archéologiques effectuées les dernières décennies ont eu comme résultat la découverte de nouveaux établissements et nécropoles médiévales. L'étude anthropologique et archéologique de ces villages médiévaux, qui appartiennent aux XVI-e -
XVII-e siècles, a eu comme résultat un important apport pour une meilleure connaissance des populations qui ont vécu pendant l'époque respective. Dans le nucleus qui a généré leur longue existence ces établissements villageois ont été influencés par 
les centres urbains des alentours ou de la zone sous montagneuse, qui ont permis des déplacements dans les deux sens par nécessité socio-économique ou fortuite.

En ce qui suit nous ferons des comparaisons entre les sujets découverts dans la necropole de Vadu Anei et ceux d'autres nécropoles médiévales, avec qui ils ont des tangences du point de vue de l'analyse anthropologique.

L'analyse s'est fait d'après les caractères métriques (la où l'état de conservation des sujets a permis cela) et morphologiques, en utilisant la technique internationale de l'école roumaine d'anthropologie. Nombreux de squelettes plus fragiles, d'enfants et de femmes, comme d'ailleurs aussi les os du visage en général étaient fragmentaires ou pulvérisés. L'absence du massif facial a fait impossible l'encadrement des respectif sujets - même en lignes générales - dans un certain type anthropologique.

Ont été découvertes et étudiés 445 sépultures pour les quelles on a constaté que le sol a eu une action corrosive sur le matériel ostéologique humain. Cet état insuffisant de conservation a abouti à l'étude en détail ou général de certains sujets, une part des aspects étant définitivement exclue. L'échantillon, découvert en 1991, a permis l'étude de détail pour la plus grande partie des individus, en comparaison avec le matériel ostéologique découvert l'année suivante, qui présentait un état de conservation impropre pour une étude anthropologique.

En mettant les squelettes par groupe d'age, on constate une grande fréquence de la mortalité infantile, des adolescents, et une rareté des sujets qui dépassaient l'age de 60 ans ( \pm 5 années). Ce fait, en liaison avec la mortalité avancée des groupes de femmes de jeune age, est un phénomène commun pour toute la période médiévale.

Sous rapport métrique et morphologique, la série de Vadu Anei est polymorphe. L'échantillon est formé de mâles robustes, avec des tendances d'oscillation de la taille moyenne vers la haute, et de femmes plus tôt graciles, ayant une stature prédominante gracile moyenne et rarement sous la moyenne. Rares sont les sujets masculins très hauts ou les femmes hautes.

Dans la norme verticale, la forme du neurocrâne est souvent ovoïde, ovoideellipsoïdal, pentagonoïde et "ronde", avec une courbure modéré ou forte chez les mâles et ovoide-sphénoïdale chez les femmes. Dans la norme occipitale les crânes ont une forme de maison, maison-bombe et rarement de bombe.

Les calottes de la série masculines, dans l'ordre de la fréquence, sont d'une longueur moyenne, ou longue, très longue et courte. Les crânes, comme hauteurs, sont moyens chez les mâles, avec quelques exceptions, hauts et très hauts, et chez les femmes ils sont prédominants moyens - ayant une hauteur modérée - et parfois d'une base hauteur.

L'indice crânien calculé de ces deux dimensions principales (là où il a été possible), $e u$-eu/g-op, nous a donné un aspect comparative avec les résultats obtenues sur des sujets découverts dans d'autres nécropoles médiévales de la Valachie, étudiées par nous (Măneşti Georgescu, 1978; Ştefănescu, 1992 şi Bârzeşti Vitan - Georgescu, 1983; Panait, Ştefănescu, 1992) et par Ioana Popovici (MăicăneştiStrăuleşti II - Popovici, 1973; Popovici, Udrescu, 1975; Panait, 1965, 1969). 
TABLEAU NO. 1

-indice crânien en pourcentages-

\begin{tabular}{|c|c|c|c|c|c|c|c|c|c|}
\hline \multicolumn{2}{|c|}{ Eu-eu/g-op 8/1 } & \multicolumn{2}{c|}{ Vadu Anei } & \multicolumn{2}{c|}{ Măneşti } & \multicolumn{2}{c|}{ Măicănești } & \multicolumn{2}{c|}{ Bârzeşti-Vitan } \\
\hline & & F & B & F & B & F & B & F & B \\
\hline $70,0-74,9$ & Dolicrâne & 12,6 & 30,6 & 3,8 & - & 7,7 & 30,8 & 6,4 & 20,6 \\
\hline $75,0-79,9$ & Mezocrâne & 58,4 & 60,0 & 68,2 & 68,0 & 61,5 & 38,5 & 62,4 & 40,8 \\
\hline $80,0-84,9$ & Brachicrâne & 29,0 & 6,4 & 26,4 & 28,2 & 30,8 & 26,9 & 28,6 & 28,2 \\
\hline $85,0-89,9$ & Ultrabrahicrâne & - & 3,0 & 1,6 & 3,8 & - & 3,8 & 2,6 & 10,4 \\
\hline
\end{tabular}

En ce qui concerne le rapport de l'hauteur du crâne à la longueur de la calotte, nous avons la suivante situation, à cause de l'indice de hauteur/longueur ( $p o-b / g-o p)$

TABLEAU NO. 2

L'indice de hauteur du crâne / sa longueur -en pourcentages-

\begin{tabular}{|l|l|l|l|l|l|l|l|l|l|}
\hline \multicolumn{2}{|c|}{ po-b/g-op } & \multicolumn{2}{l|}{ Vadu-Anei } & \multicolumn{2}{l|}{ Măneşti } & \multicolumn{2}{l|}{ Măicăneşti } & \multicolumn{2}{l|}{ Bârzeşti-Vitan } \\
\hline \multicolumn{2}{|c|}{$20 / 1$} & F & B & F & B. & F & B & F & B \\
\hline x-57,9 & Camaecrâne & 3,6 & 2,8 & - & - & 71,5 & 53,8 & 68,4 & 62,6 \\
\hline $58-62,9$ & Ortocrâne & 58,4 & 63,2 & 56,4 & 43,6 & 71,5 & 53,8 & 68,4 & 62,6 \\
\hline $63-x$ & Hipsicrâne & 38,0 & 34,0 & 43,6 & 56,4 & 29,5 & 46,2 & 31,6 & 37,4 \\
\hline
\end{tabular}

TABLEAU NO. 3

L'indice de l'hauteur du crâne / son épaisseur -en pourcentages-

\begin{tabular}{|l|l|c|c|c|c|c|c|c|c|}
\hline \multicolumn{2}{|c|}{ Po-be/eu-eu } & \multicolumn{2}{c|}{ Vadu-Anei } & \multicolumn{2}{c|}{ Măneşti } & \multicolumn{2}{c|}{ Măicăneşti } & \multicolumn{3}{c|}{ Bârzeşti-Vitan } \\
\hline \multicolumn{2}{|c|}{$20 / 8$} & F & B & F & B & F & B & F & B \\
\hline x-79,9 & Tapeinocrâne & 86,2 & 42,8 & 68,0 & 56 & 86,6 & 46,1 & 66,8 & 48,8 \\
\hline $80,0-85,9$ & Metriocrâne & 12,8 & 38,6 & 20,8 & 44 & 6,6 & 46,1 & 20,2 & 34,2 \\
\hline $86,0-x$ & Acrocrâne & 1,0 & 18,6 & 11,2 & - & 6,7 & 7,8 & 13,0 & 17,0 \\
\hline
\end{tabular}

Le rapport de l'hauteur du crâne à la largeur nous donne les suivantes données de l'indice de l'hauteur sur la largeur du crâne (pob/eu-eu).

Le facial, pour les cas qu'il s'est gardé, est assez de variable, comme d'ailleurs aussi dans les autres nécropoles de la Plaine Roumaine, avec des petites exceptions que nous allons faire mention quand nous parlerons sur l'aspect de la structure de base et de la typologie.

Le frontal présente des valeurs qui permettent l'encadrement des sujets dans toutes les catégories, de très étroites aux très larges, les indices frontaux en les caractérisants comme des fronts bombé-intermédiaires, metriométopes, sténométopes et eurimétopes. L'indice facial est rarement hipereuriprosope et 
mezoprosope mais souvent fréquent, et parfois leptoprosope.

Chez les femmes le relief supra-orbiter est modéré, vers des formes légèrement tracées et souvent essuyées, en comparaison avec les mâles, qui en grande partie ont le relief fortement glabellé et très fort en relief.

Les orbites sont mesoconques vers hipsiconques, encadrant, pour les cas qu'elles se sont gardées, des nez leptorins, mezorins et chamerhins. L'indice Flower est orthogoné, avec des petites exceptions.

Les mandibules présentent chez les femmes des formes plus graciles, avec des gonions effacés et des petits mentons, en général arrondis, et chez les mâles, les gonions sont retroussés fortement ou modéré au dehors de la branche verticale, ayant des mentons fort en relief chez les formes massifs, ou modéré autrement.

Les pariétaux ont des légers arrondissements chez les femmes, jusqu'au moyen-bombé, les sujets masculins présentant des arrondissements moyens, vers un fort arrondissement parfois. L'occipital se présente ayant des divers grades de courbure, planoccipitale en trois cas, large et moyen chez les mâles, moyen chez les femmes. Le relief nucal a des formes fort en relief chez les mâles et faible couturé chez les femmes, avec des courtes apophyses, au relief effacé chez les femmes et fort couturé et beaucoup plus hautes, de grosseurs divers chez les mâles.

$\mathrm{Si}$ aux sujets de la nécropole de Măicăneşti-Străuleşti le diformisme sexuel est moins accentué dans la portion supérieure du visage, chez les mâles-mezen et chez les femmes-eurien, que surtout dans les limites de la variabilité individuelle (eurien-mezen) pour toute la série, chez les sujets de la nécropole de Vadu Anei la difformité sexuel est prononcé aux étages supérieure et inférieure du visage chez les mâles (spécialement) et se manifeste plus prégnant dans la construction du squelette postcrânien, les femmes étant, en générale, plus graciles.

Une note commune aux séries de Măicăneşti et Vadu Anei est la présence d'un prognathisme alvéolaire et d'une prodentia (rencontré à Grozăveşti - Panait, Ştefănescu,
1975; Georgescu, 1976 - et Oltenița-Renie Georgescu, 1978; Şerbănescu, 1978). De même, nous signalons la grande fréquence des caries dentaires-59,0\% à Măneşti et $64,3 \%$ à Vadu Anei - que l'usure dentaire-56,8\% à Măneşti et $68,2 \%$ à Vadu Anei. Ce fait peut être mis en liaison avec les conditions de vie des groupes humains étudiés, avec le type d'alimentation, avec la perioade de fertilité des femmes.

La où l'état de conservation du squelette post crânien l'a permit, la taille a été calculée d'après la méthode de Manouvrier (et avec la correction respective, donnée de même par lui); à Măneşti, la taille se situe entre les limites 158,0 cm. $-164,4 \mathrm{~cm}$ chez les femmes et entre 163,2 cm.- $178,3 \mathrm{~cm}$. chez les mâles, pour les sujets des XIV-e - XV-e siècles, et chez les sujets des XVI-e - XVII-e siècles nous avons seulement des petites différences à la limite supérieur, autant chez les femmes $(158,2 \mathrm{~cm}$. $166,2 \mathrm{~cm})$ que chez les mâles $(164,6 \mathrm{~cm}$. -179 , $6 \mathrm{~cm})$.

En ce qui concerne la nécropole de Măicăneşti-Străuleşti, ayant la même datation, nous avons la suivante situation: la taille s'encadre une seule fois chez les mâles et deux fois chez les femmes dans la catégorie des très petites tailles; plusieurs cas chez les mâles sont groupés dans les catégories des tailles petites, moyennes et très hautes, et chez les femmes tous les sujets se sont groupé dans la catégorie des tailles moyennes.

A Vadu Anei, la situation se présente ainsi en ce qui concerne la taille des sujets qui on pu être mesurés: la plus grande partie des mâles sont robustes, avec une tendance d'oscillation de la taille des valeurs moyennes vers hautes $(168,2 \mathrm{~cm} .-179,2 \mathrm{~cm}$.), avec des rares exceptions pour les cas très hauts (182,0 cm. - 184,6 cm); en ce qui concerne les femmes, elles sont graciles, la gracilité moyenne et sous-moyenne $(154,0 \mathrm{~cm}$. $-158,6$ $\mathrm{cm}$ ), mais on a observer aussi quelques cas de grande hauteur $(159,2 \mathrm{~cm} .-168,0 \mathrm{~cm}$.), un peu robustes.

L'étude morfo-fonctionelle a relevé la présence dès fortes impressions musculaires au niveau des insertions musculaires, fait qui nous permet d'avancer l'hypothèse que les sujets de Vadu Anei étaient assez d'homogènes comme groupe, ayant une intense activité, 
caractéristique pour la population agricole de la zone étudié, aspect que nous avons surpris et chez les sujets des trois nécropoles de Măneşti. Nous ne pouvons pas faire des références pour les sujets découverts dans la nécropole de Bârzeşti -Vitan, qui ne bénéficie pas d'une étude complète et ni pour les sujets de Măicăneşti, où on n'a pas fait des pareilles études. Nous faisons la mention que nous avons pu établir un diagnostique, autant pour les sujets des nécropoles de Măneşti que de VaduAnei, la présence de la maladie rhumatismale, avec ses évolutions déformantes, à cause du milieu humide, des fractures consolidés d'une manière défectueuse et déformante à cause des pratiques empiriques, ou en certain cas sans aucune intervention réparatrice.

Les aspects de l'étude dento-maxilaire seront abordés séparément comme d'ailleurs aussi ceux démographique.

En ce qui concerne l'encadrement typologique par séries, avec certaines réserves d'un cas à l'autre, comme suite de l'etat de conservation:

- pour le groupe des sujets de Măneşti (Georgescu, 1978) nous pouvons dire que ceuxci appartiennent au type europoïde avec une prédominance de l'élément méditerranéen;

- en ce qui concerne les sujets étudiés de la série Măicăneşti-Străuleşti, après que nous avons précisé leur appartenance à l'origine europoïde de la population, on souligne que les "méditerranéens représenteraient l'élément caractéristique de la région" (Popovici, 1973), confirmant ainsi encore une fois la caractéristique du fond méditerranéen dans la Plaine du Danube (on ne doit pas oublier que les méditerranéens ont constitué l'élément prédominant dans la plaine nord-danubienne depuis le néolithique);

- la meme situation, avec des petites exceptions, nous l'avons à Bârzeşti-Vitan (Georgescu, 1983);

- les séries de Grozăveşti (Georgescu, 1976) et Oltenița Renie (Georgescu, 1978) sont homogènes sous le rapport des caractères europoïdes, présentant, dans l'ordre de la fréquence, les suivants types : méditerranoïde, méditerranoïde avec des éléments nordiques, méditerranoïde avec des éléments est- europoïdes, nordique avec des éléments esteuropoïdes, proto-europoïdes;

- les sujets de la nécropole de Vadu Anei ont des encadrements tout proches de ceux de la nécropole de Radovanu (Popovici, Georgescu, 1975; Georgescu, 1986), plus précis avec ceux $\mathrm{du}$ groupe I (qui ont des certaines caractéristiques méditerranoïdes) et du groupe II (qui présente certains caractères semblables aux nordiques, mais aussi aux méditerranoïdes robustes). Le groupe II de Radovanu se détache des autres groupes des inhumés de la même nécropole et des autres nécropoles mentionnées plus haut comme type dans le sens que les sujets présentent certaines caractéristiques Cromagnoïdes (B), mais aussi dynaroïdes (Popovici, Georgescu, 1975; Georgescu, 1986); Radovanu a des tangences avec la serie tardive de Cernica (Botezatu, Ştefănescu, 1975), dans son dernier groupe. Une extension de la recherche de terrain dans la nécropole de Radovanu pourrait clarifié beaucoup de situation. En ce qui concerne l'existence des sujets brachycrâne, ce fait peut être une conséquence des infiltrations dans la zone des groupes brachycrânes, avec l'exception des groupes de brachycrânes de Măneşti et Bârzeşti-Vitan, qui, pour le moment, nous supposons qu'ils serraient le résultat $\mathrm{du}$ processus de brachycéphalisation par évolution sur place. Mais cette discutions reste ouverte pour les recherches futurs, sur les quelles nous allons insister spécialement, parce que nous avons besoin de sérieuses preuves.

Un autre problème relevé par le groupe humain de Vadu Anei est celui de la déposition des inhumés: ils sont déposés en decubit dorsal avec les membres supérieures en flexion sur le bassin, sur la ceinture ou avec les deux membres supérieures (ou seulement avec l'un d'eux) sur la clavicule, et parfois sur le sterne. Les représentants de ces modes d'inhumation ont des caractères communs - pouvant être des différences régionales de conception. Ce fait, comme aussi à Măicăneşti-Străuleşti, n'exclu pas la possibilité des origines ethniques différentes, en connaissant le fait que les éléments méditerranéens ont une très large zone de diffusion.

Autant Ioana Popovici, dans son etude sur le materiel decouvert à Măicăneşti- 
Străuleşti, que nous aussi, pendant les recherches de Mănești, Bârzeşti-Vitan, Grozăveşti, Oltenița-Renie, Ceptura (Georgescu, Lichiardopol, 1986), Vadu Anei, nous avons remarqué comme une caractéristique pour toute la population étudiée le mezoprognatisme alvéolaire et la prodentia (surtout souvent aux sujets masculins). Nous supposons (jusqu'à des nouvelles preuves) que ces éléments ont été apportés par des individus mezoprognés et gardés dans le circuit endogame, ou nous le supposons qu'ils sont eu une apparition "indépendante" et ils se sont gardé de même comme conséquence de l'endogamie. Cette caractéristique nous l'avons en une proportion assez grande dans la Plaine du Danube.

La très grande fréquence de la mortalité infantile, (infans I), des adolescents et des femmes du groupe d'age jeune ne peut être par hasard. Nous supposons que l'assemblement des enfants dans certaines zones peut être la conséquence d'une épidémie, qui bien sur a affecté non seulement ce groupe d'age, mais aussi celui des femmes jeunes.

Parce que pendant la période étudié la maturité se produisait plus tôt, nous avons étudié la mortalité et nous avons fait des références à l'espérance de vie pendant un certain intervalle d'age, au quel ont fait des références aussi d'autres anthropologues, comme il suit (à voir le Tableau no. 4 où on trouvent les nécropoles qui figurent dans l'étude comparative):

\begin{tabular}{|l|l|l|l|l|}
\hline Nécropole & Nr. cas & Siècle & Etude anthropologique & $\begin{array}{l}\text { Etude } \\
\text { archéologique }\end{array}$ \\
\hline Bârzeşti-Vitan & 204 & XVI & L. Georgescu & $\begin{array}{l}\text { Panait I. Panait } \\
\text { A. Ştefănescu }\end{array}$ \\
\hline Cernica & 87 & XV-XVIII & $\begin{array}{l}\text { D. Botezatu } \\
\text { Gh. Ştefănescu }\end{array}$ & N. Constantinescu \\
\hline Ceptura & 67 & XVI & L. Georgescu & D. Lichiardopol \\
\hline Bragadiru-Zimnicea & 52 & XVI & I. Popovici & Gh. Bichir \\
\hline Grozăveşti & 22 & XVI & L. Georgescu & A. Ştefănescu \\
\hline Măneşti & 272 & XVI-XVII & L. Georgescu & A. Ştefănescu \\
\hline Măicăneşti-Străuleşti & 258 & XVI & I. Popovici & Panait I. Panait \\
\hline Oltenița-Renie & 24 & XVI & L. Georgescu & D. Şerbănescu \\
\hline Radovanu & 164 & XVI-XVIII & I. Popovici, L. Georgescu & M. Comşa \\
\hline Vadu Anei & 450 & XVI-XVII & L. Georgescu & $\begin{array}{l}\text { A. Păunescu } \\
\text { S. E. Teodor }\end{array}$ \\
\hline
\end{tabular}

- L'espérance de vie dans l'intervalle infans I à Bârzești-Vitan est de 28,6 années, à Bragadiru- Zimnicea (Popovici 1969) de 32,1 années, à Cernica de 32,4 années, à Ceptura de 30,2 années. A Măneşti, dans les séries réunîtes, l'espérance de vie pour ce intervalle est de 32,6 années, et à Măicăneşti-Străuleşti II de 29,0 années. Les sujets de Olteniţa-Renie s'approchent de ceux de Grozăveşti, ayant une espérance de vie de 30,2 années. Le matériel de Radovanu, analysé de nouveau par nous pour ce problème, au même intervalle d'age (infans I) nous a donné des valeurs oscillantes entre 22,0 années et 23,2 années. En ce qui concerne les sujets de Vadu Anei, ils ont une espérance de vie d'approximativement 32,2 années.

Dans le deuxième intervalle de mortalité élevé, 20-30 années, l'espérance de vie par nécropoles s'encadre ainsi: les sujets de la nécropole de Bârzeşti-Vitan ont une espérance de vie de 23,2 années; ceux de BragadiruZimnicea ont une espérance de vie de 20,6 années; le groupe de Cernica se fixe à 23,4 années. En ce qui concerne les séries réunîtes de Măneşti nous avons des valeurs d'approximativement 22,6 années, et à Măicăneşti-Străuleşti II de 22,0 années; les groupes humains de Grozăveşti et Oltenița-Renie ont une espérance de vie d'approximativement 
20,8 années. Dans le même intervalle d'age, 2030 années, le groupe de Radovanu présente une espérance de vie de 22,4 années. Les sujets de Vadu Anei présente une espérance de vie d'approximativement 22,2 années.

La distribution de la population par groupes d'age nous donne d'une manière plus détaillée la structure d'une population ou d'un groupe humain. Ainsi nous nous rendons compte si la population est formé surtout d'éléments jeunes et adultes et quel est le pourcentage d'éléments vieux, inversant les résultats de la mortalité constatée, parce que les inhumés ne représente pas la totalité des individus qui ont vécu dans l'aire étudié.

A l'aide des tabelles de mortalité pour chaque groupe d'age ont été calculés la moyenne de vie, la probabilité de survivre et les résultats de la recherche démographique roumaine s'encadrant dans "le tableau du monde médiévale" (Acsady, Nemeskery, 1970). L'objet de la démographie est l'homme regardé dans la collectivité et non isolé, en étroite interdépendance avec le milieu ambiant. L'étude de la démographie est fort important, parce que nous pouvons nous rendre compte en n'importe quel moment de la structure et de la dynamique d'une collectivité, surtout des tendances des phénomènes démographiques qui conduisent aux transformations dans la structure de la population (Pascu, 1970; Matei, 1973, Nemeskery, 1970; Pescaru 1968). Les phénomènes démographiques doivent être regardé en étroites liaisons avec l'état économique-sociale.

\section{BIBLIOGRAPHIE}

Acsady, Nemeskery, 1970 - Acsady G., Nemeskery, History of human life span and mortality, Budapeste, 1970.

Botezatu, Ştefănescu, 1975 - D.Botezatu, Gh. Ştefănescu, Caracterizarea antropologică a cimitirului feudal de la Cernica-Sec.XVI-XVIII, SCA, IX, 1975.

Georgescu, 1978 - Laurenția Georgescu, Necropolele feudale de la Măneşti-Buftea, Ilfov-File de istorie, 1978. Georgescu, Lichiardopol, 1986 - L. Georgescu, D. Lichiardopol, Necropola medievală de la Ceptura. Date arheologice şi antropologic, Anuarul Muzeului de Istorie şi Arheologie, Ploieşti, 1986.

Matei, 1973 - Vlad D. Matei, Colonizarea Rurală În Țara Românească şi Moldova În Sec. XV-XVIII, Bucurest, 1973.

Panait, 1965 - Panait I. Panait, Şantierul arheologic Băneasa-Străuleşti. Aşezarea feudală de la Străuleşti-Măicăneşti, CAB, II, Bucurest, 1965.

Panait, Ştefănescu, 1975 - Panait I. Panait, A. Ştefănescu, Relațiile oraşului Bucureşti cu satele invecinate, Muzeul Naţional, II, Bucurest, 1975.

Pascu, 1970 - Şt. Pascu, Demografia istorică, în vol. Populație şi societate, Cluj, 1970.

Pescaru, 1968 - Al. Pescaru, Elemente de demografie, Bucurest, 1968.

Popovici, 1969 - Ioana Popovici, Necropola feudală de la Bragadiru-Zimnicea (sec. XVI). Observații antropologice, SCA, tom.6, nr.2, 1969.

Popovici, 1973 - Ioana Popovici, Cimitirele de la Străuleşti. Analiză demografică, SCA, tom 10, nr.1, 1973.

Popovici, Georgescu, 1975 - Ioana Popovici, Laurenția Georgescu, Contribuții antropologice la studiul populației feudale. Cimitirul de la Radovanu, SCA, tom 12, 1975.

Popovici, Udrescu, 1975 - Ioana Popovici, M. Şt. Udrescu, Date comparative asupra unor populații medievale din Câmpia Română, SCA, tom.9, 1975.

Şerbănescu, Georgescu, 1978 - D. Şerbănescu, Necropola feudală de la Oltenița Renie, Ilfov-File de istorie, 1978.

\section{ABREVIATIONS}

CAB Cercetări Arheologice în Bucureşti

SCA Studii şi Cercetări de Antropologie 